\title{
MODIFICACIÓN ÁCIDA DEL CARBÓN ACTIVADO Y LA INFLUENCIA DEL GRUPO SUSTITUYENTE EN LA ADSORCIÓN DE COMPUESTOS FENÓLICOS
}

\author{
Edwin Javier Aylas Orejón ${ }^{1}$, Gino Picasso Escobar², María del Rosario Sun Kou ${ }^{1}$
}

\begin{abstract}
RESUMEN
En este trabajo, se ha estudiado la influencia de grupos funcionales oxigenados en la capacidad adsortiva de un carbón activado (CA) preparado a base de semillas de aguaje (Mauritia flexuosa L.F.) por activación química con ácido fosfórico. Luego el carbón activado fue oxidado con ácido nítrico y se comparó la capacidad adsortiva del carbón activado (CA) y del carbón activado oxidado (ACA) en la adsorción de compuestos fenólicos: fenol, 4-clorofenol y 4-nitrofenol. La caracterización de ambos carbones activados se realizó a través de las técnicas de adsorción-desorción de nitrógeno, titulación Boehm, microscopía electrónica de barrido (SEM) y espectroscopía FTIR. La muestra oxidada (ACA) mostró un aumento en el área microporosa de $756 \mathrm{~m}^{2} / \mathrm{g}$ a $1272 \mathrm{~m}^{2} / \mathrm{g}$, de igual manera los grupos funcionales oxigenados totales aumentó de 4,22 $\mathrm{mmolH}^{+}$. $\mathrm{g}^{-1}$ a $4,82 \mathrm{mmolH}^{+} . \mathrm{g}^{-1}$ y el punto de carga cero (pHPZC) se redujo de 2,450 a 2,014. Se encontró que hay una fuerte influencia del grupo sustituyente en la adsorción del carbón. El aumento de los grupos funcionales oxigenados disminuyó la capacidad adsortiva de los carbones activados. La capacidad de adsorción tanto del CA y ACA mostró el siguiente orden: 4-nitrofenol > 4-clorofenol > fenol. Se encontró que el modelo de pseudo segundo orden correlacionó mejor los datos experimentales.
\end{abstract}

Palabras clave: Carbón activado, oxidación, compuestos fenólicos

\section{ACID MODIFICATION OF ACTIVATED CARBONS AND THE INFLUENCE OF SUBSTITUENT GROUP IN THE ADSORTION OF PHENOLIC COMPOUNDS}

\begin{abstract}
In this work, it has been studied the influence of oxygenated functional groups on adsorption property of an activated carbon (CA), which was prepared from aguaje seeds (Mauritia flexuosa L. F.) by a chemical activation with phosphoric acid. Later the activated carbon was oxidized with nitric acid. The adsorptive capacity of phenolic compounds such as phenol, 4-chlorophenol and 4-nitrophenol were compared using both of the adsorbents, the activated

\footnotetext{
${ }^{1}$ Sección Química - Departamento de Ciencias. Pontificia Universidad Católica del Perú. Av. Universitaria 1801. Lima 32. San Miguel. Lima-Perú. *Email: msun@pucp.edu.pe.

${ }^{2}$ Laboratorio de Investigación de Fisicoquímica (LABINFIS). Facultad de Ciencias. Universidad Nacional de Ingeniería. Av. Túpac Amaru 210. Rímac. Lima-Perú
} 
carbon (CA) and the oxidized activated carbon (ACA). The characterizations of both activated carbons were analyzed by nitrogen adsorption measurements, Boehm titration, scanning electron microscopy (SEM) and FTIR spectroscopy. The oxidized sample (ACA) showed an increase in the microporous area from $756 \mathrm{~m}^{2} / \mathrm{g}$ to $1272 \mathrm{~m}^{2} / \mathrm{g}$, likewise ACA presented an increase in the concentration of oxygenated functional groups from $4,22 \mathrm{mmolH}^{+} . \mathrm{g}^{-1}$ to $4,82 \mathrm{mmolH}^{+} \cdot \mathrm{g}^{-1}$. In contrast, the point of zero charge (pHPZC) decreased from 2,450 to 2,014. This suggested that there is a strong influence of the substituent group on the carbon adsorption. The increase in the oxygenated functional groups originated the decrease in the adsorptive capacity of activated carbons. The adsorption capacity of both carbons CA and ACA showed the following order: 4-nitrophenol $>4$-chlorophenol $>$ phenol. It was found that the pseudo second-order model was the best fit model for the experimental data.

Key words: Activated carbon, oxidation, phenolic compounds

\section{INTRODUCCIÓN}

Los carbones activados son utilizados en el tratamiento de aguas ${ }^{1}$ con la finalidad de adsorber, entre otros contaminantes, los metales pesados. Esto es debido a dos factores que presentan: la textura y la química superficial. Se han realizado trabajos de tratamientos específicos ${ }^{2}$ con la finalidad de mejorar estas propiedades y así aumentar la versatilidad de su empleo. Uno de los tratamientos más utilizados es la oxidación ácida de los carbones activados con ácido nítrico, pues con ello se aumenta la cantidad de grupos oxigenados en la superficie del carbón, como se ha comprobado en algunos trabajos ${ }^{3,4} \mathrm{y}$ ha sido un factor determinante en la adsorción de metales.

Por otro lado, se ha encontrado que el aumento de los grupos oxigenados en la superficie del carbón ocasiona la disminución de la capacidad adsortiva de los carbones hacia los contaminantes orgánicos, especialmente los derivados fenólicos ${ }^{5}$. Por esta razón, el presente trabajo se ha orientado al estudio de la influencia que la oxidación ácida tiene sobre la adsorción de compuestos fenólicos con diferentes grupos sustituyentes y al análisis de la relación que estos tienen en el proceso de adsorción usando carbones activados.

Coughlin y Ezra ${ }^{6}$ propusieron que cuando el fenol se adsorbe, a partir de disoluciones diluidas, lo hace de forma que su anillo aromático queda paralelo a las láminas de grafeno de la superficie del carbón. De esta forma, la adsorción se produce mediante interacciones electrostáticas entre los electrones $\pi$ del anillo aromático y los electrones $\pi$ de las láminas de grafeno.

La presencia de grupos sustituyentes en el anillo aromático produce cambios en la solubilidad de los compuestos fenólicos, en el carácter ácido y nucleofílico. Así, el grupo (-OH) es considerado fuertemente activante, el átomo de cloro $(-\mathrm{Cl})$ es débilmente desactivante, mientras que el grupo $\left(-\mathrm{NO}_{2}\right)$ es fuertemente desactivante. Esto causa que la afinidad relativa entre el carbón y el compuesto fenólico varíe considerablemente. 
Para poder corroborar esta relación entre los grupos sustituyentes y su retención en la superficie del adsorbente, se ha estudiado la adsorción del fenol, 4-clorofenol y 4-nitrofenol, tanto en carbón activado, como en carbón activado oxidado con ácido nítrico.

\title{
PARTE EXPERIMENTAL
}

\begin{abstract}
A) Preparación de carbón activado (CA)
El carbón activado (CA) se preparó a partir de semillas de aguaje (Mauritia flexuosa L.F.) por activación química con ácido fosfórico usado como agente activante ${ }^{7}$. Inicialmente, el precursor fue reducido de tamaño en forma granular y se tamizó hasta obtener un tamaño uniforme de partícula $(3-5 \mathrm{~mm})$. La impregnación se llevó a cabo a $85^{\circ} \mathrm{C}$ durante $2 \mathrm{~h}$ con soluciones de ácido fosfórico con una relación de impregnación de $0,75 \mathrm{gH}_{3} \mathrm{PO}_{4}$ /gprecursor. Después de la impregnación, las muestras se carbonizaron a $600{ }^{\circ} \mathrm{C}$ con una velocidad de calentamiento de $8^{\circ} \mathrm{C} / \mathrm{min}$ y con un flujo de nitrógeno de $250 \mathrm{~mL} / \mathrm{min}$. Finalmente, la muestra se lavó con agua destilada hasta obtener un $\mathrm{pH}=5$, se secó a $100^{\circ} \mathrm{C}$ y tamizó hasta $0,25 \mathrm{~mm}$ ( ${ }^{\circ} 60$-malla, ASTM).
\end{abstract}

\section{B) Oxidación del carbón activado (ACA) con ácido nítrico}

Para el tratamiento de oxidación con ácido nítrico $\left(\mathrm{HNO}_{3}, 1 \mathrm{M}\right)$ se utilizó $2 \mathrm{~g}$ de CA, que se mezcló con $160 \mathrm{~mL}$ de la solución ácida durante 6 horas a $65^{\circ} \mathrm{C}$; a continuación, la mezcla se filtró y el carbón se lavó con agua destilada a fin de eliminar los restos de ácido. Por último, la muestra oxidada se secó a $100^{\circ} \mathrm{C}$ durante $24 \mathrm{~h}$. La muestra de carbón activado oxidado se denominó ACA.

\section{C) Caracterización de los carbones activados}

La caracterización textural, química y morfológica de los carbones activados con y sin oxidación, se realizó utilizando las siguientes técnicas:

\section{1) Adsorción-desorción de $\mathrm{N}_{2}$}

El área superficial y el volumen de poros de las muestras de carbón activado se evaluaron por adsorción-desorción de $\mathrm{N}_{2}$ a $77 \mathrm{~K}$ en el rango de presiones relativas entre 0,005 a 1, en un equipo Gemini VII modelo 2390 t; el área superficial específica (SBET), se calculó mediante el modelo Brunauer-Emmett-Teller (BET). El volumen y área microporosa se determinó utilizando el método $t$-plot.

2) Titulación Boehm- determinación de los grupos ácidos superficiales.

Para la determinación de los grupos ácidos superficiales por Titulación Boehm se preparó una serie de muestras, en cada una se pesó $0,02 \mathrm{~g}$ de carbón activado y se mezcló con $20 \mathrm{~mL}$ de soluciones de hidróxido de sodio $(\mathrm{NaOH})$, carbonato de sodio $\left(\mathrm{Na}_{2} \mathrm{CO}_{3}\right)$ y bicarbonato de sodio $\left(\mathrm{NaHCO}_{3}\right)$, cada una de concentración $0,05 \mathrm{M}$, respectivamente. Las soluciones se mantuvieron a temperatura ambiente, con agitación constante durante $24 \mathrm{~h}$. Se titularon alícuotas de $5 \mathrm{~mL}$ de cada una de las soluciones con $\mathrm{HCl}$ de 0,05 M. 
3) Espectroscopia infrarroja (FTIR)

La espectroscopia infrarroja permitió determinar la presencia de los diferentes grupos funcionales, los cuales poseen un pico característico en las diferentes zonas del infrarrojo. Previo al ensayo, la muestra a analizar fue secada en la estufa a $100^{\circ} \mathrm{C}$ por $24 \mathrm{~h}$. Se pesó 0,01 $\mathrm{g}$ de la muestra la cual fue pulverizada y mezclada con $0,09 \mathrm{~g}$ de $\mathrm{KBr}(\mathrm{s})$ en un mortero de ágata. La mezcla fue comprimida en una prensa hasta obtener una pastilla fina, la cual fue colocada en el espectrómetro Perkin Elmer modelo Spectrum 100 FTIR y fue analizada en el rango espectral de $4000-400 \mathrm{~cm}^{-1}$.

4) Determinación del punto de carga cero (pHPZC)

Para determinar el punto de carga cero, se preparó una serie de muestras, en cada una se pesó aproximadamente $500 \mathrm{mg}$ de carbón activado y se mezcló con $20 \mathrm{~mL}$ de soluciones ácidas y básicas durante $24 \mathrm{~h}$, se controló que el $\mathrm{pH}$ fuese igual a 2, 3, 4, 6 y 8 . Para regular el $\mathrm{pH}$, se utilizó $\mathrm{HCl}$ para las soluciones ácidas y $\mathrm{NaOH}$ para las soluciones básicas. Luego, la muestra se filtró y se determinó su pH utilizando un pHmetro WTW modelo 537 y un electrodo de $\mathrm{pH}$ con electrolitos líquidos de $\mathrm{Ag} / \mathrm{AgCl}_{2}$ (marca Sentix modelo 81). El pHPZC se obtuvo al graficar los valores de $\Delta \mathrm{pH}$ versus $\mathrm{pH}$ inicial.

5) Microscopía electrónica de barrido (SEM)

El análisis morfológico se realizó a través de la microscopía electrónica de barrido (SEM). Los ensayos se realizaron en un microscopio electrónico de barrido FEI Quanta 650. Las micrografías SEM fueron tomadas con tres tipos de sistemas de detección: electrones secundarios (SE), electrones retrodispersos (BSE) y detección mixta (MIX).

Previo al ensayo, la muestra a analizar se secó a $80{ }^{\circ} \mathrm{C}$ en la estufa durante $16 \mathrm{~h}$ para eliminar cualquier traza de humedad. Luego, se esparció una pequeña cantidad de la muestra en una oblea de carbono, hasta tener una superficie cubierta uniformemente. La oblea fue llevada al microscopio para el análisis morfológico (SEM). Las micrografías se obtuvieron con aumentos de 400X.

\section{D) Proceso de adsorción}

En un trabajo previo se determinó el $\mathrm{pH}$ y la masa de adsorbente óptimo para la eliminación del fenol, 4-clorofenol y 4-nitrofenol con carbones activados preparados a partir de semillas de aguaje ${ }^{8}$. Previo a los ensayos de adsorción de los compuestos fenólicos, se determinó la curva de calibración con soluciones de 5, 10, 20, 40 y 60 ppm además de un blanco.

Los ensayos se realizaron por duplicado en un sistema por lotes y se determinó la concentración de los compuestos fenólicos al inicio y después de transcurrido un tiempo de contacto predeterminado hasta alcanzar la condición de equilibrio. Se analizó la señal del fenol a $270 \mathrm{~nm}$, el 4-clorofenol a $280 \mathrm{~nm}$ y el 4-nitrofenol a $317 \mathrm{~nm}$, utilizando un espectrofotómetro UV-Visible Perkin Elmer modelo Lambda 2 y se determinó la capacidad de adsorción (qt) mediante la siguiente ecuación: 


$$
q_{t}=\frac{V\left(C_{0}-C_{1}\right)}{m}
$$

Donde, qt es la capacidad de adsorción del compuesto fenólico (mg comp.fenólico/gCA), $\mathrm{C}_{\mathrm{o}}$ y $\mathrm{C} t$ son las concentraciones inicial y después de un tiempo $\mathrm{t}$, respectivamente (mg comp. fenólico/L); V es el volumen de solución (L) y m es el peso de carbón activado (g).

1) Modelo cinético de pseudo primer orden

El modelo de pseudo primer orden define que la adsorción se da por la diferencia entre la capacidad de adsorción en el equilibrio (qe) y el obtenido a un tiempo $\mathrm{t}$ (qt). La ecuación linealizada se representa por:

$$
\ln \left(\mathrm{q}_{0}-\mathrm{q}_{\mathrm{t}}\right)=\ln \mathrm{q}_{\mathrm{e}}-k_{1} \mathrm{t}
$$

Donde:

$\mathrm{q}_{\mathrm{e}}$ : capacidad de adsorción del adsorbato en la condición de equilibrio ( $\left.\mathrm{mg} / \mathrm{g}\right)$

qt: capacidad de adsorción del adsorbato a un tiempo $\mathrm{t}(\mathrm{mg} / \mathrm{g})$

$k 1$ : constante de velocidad del modelo de pseudo primer orden $\left(\mathrm{min}^{-1}\right)$

$\mathrm{t}$ : tiempo empleado en el ensayo de adsorción (min).

2) Modelo cinético de pseudo segundo orden

El modelo de pseudo segundo orden define el proceso considerando predominantemente una adsorción química del adsorbato sobre la superficie de los carbones activados. La forma linealizada de este modelo es:

$$
\frac{t}{q_{t}}=\frac{1}{k_{2} q_{e}^{2}}+\frac{1}{q_{e}} t
$$

Donde:

qt: capacidad de adsorción del adsorbato a un tiempo $\mathrm{t}(\mathrm{mg} / \mathrm{g})$

t: tiempo empleado en el ensayo de adsorción (min)

$\mathrm{q}_{\mathrm{e}}$ : capacidad de adsorción del adsorbato en el equilibrio $(\mathrm{mg} / \mathrm{g})$

k2: constante de velocidad del modelo de pseudo segundo orden (g/mg.min)

Se calculó la desviación estándar normalizada entre los valores experimentales y los valores calculados con los modelos cinéticos, con la finalidad de evaluar el mejor ajuste del modelo, la desviación estándar se calculó mediante la ecuación:

$$
\Delta \mathrm{q}_{\mathrm{e}}(\%)=100 \sqrt{\frac{\sum\left[\mathrm{q}_{\mathrm{e}(\exp )}-\mathrm{q}_{\mathrm{e}(\text { pro })} / \mathrm{q}_{\mathrm{e}(\exp )}\right]^{2}}{\mathrm{~N}-1}}
$$


Donde:

$\mathrm{q}_{\mathrm{e}}(\exp )$ : valor experimental $(\mathrm{mg} / \mathrm{g})$

$\mathrm{q}_{\mathrm{e}}$ (pro) : valor correspondiente al $\mathrm{q}_{\mathrm{e}}(\mathrm{mg} / \mathrm{g})$ pronosticado de acuerdo al modelo,

$\mathrm{N} \quad$ : número de mediciones.

El menor valor obtenido de la desviación estándar normalizada indica el mejor ajuste de los resultados experimentales.

\section{RESULTADOS Y DISCUSIÓN}

La tabla 1 muestra las características texturales del carbón activado (CA) y del carbón activado oxidado (ACA). La concentración del ácido nítrico $(1 \mathrm{M})$, temperatura $\left(65^{\circ} \mathrm{C}\right)$ y tiempo de oxidación (6h), aplicados en la preparación de la muestra ACA se mantuvieron constantes a fin de mantener la superficie del carbón sin alteración. Los resultados muestran un aumento del área superficial y de la microporosidad del carbón con el proceso de oxidación (tabla 1). El cambio de la estructura porosa se atribuye a la mayor formación y posible reestructuración de los grupos ácidos oxigenados, como se observó por los resultados de Titulación Boehm. Similares resultados fueron encontrados por Rodríguez et al. ${ }^{9}$ cuando oxidaron carbón activado con ácido nítrico y lo utilizaron en la adsorción de niquel. Chun et al. ${ }^{10}$ mostraron que el tratamiento con ácido nítrico concentrado y con temperaturas elevadas producen deterioros del área superficial del adsorbente. Este efecto también fue reportado por Ríos et al. ${ }^{11}$ y Aburub y Wirster ${ }^{12}$, quienes utilizaron ácido nítrico concentrado y tiempos de tratamiento de 24 a $38 \mathrm{~h}$, ellos reportaron una reducción del área superficial del material adsorbente en $6,5 \%$ y $33,7 \%$, respectivamente.

Tabla 1. Características texturales de los carbones: CA y ACA

\begin{tabular}{cccc}
\hline Muestra & $\begin{array}{c}\text { Área Total } \\
\left(\mathbf{m}^{\mathbf{2}} / \mathbf{g}\right)\end{array}$ & $\begin{array}{c}\text { Área mesoporosa } \\
\left(\mathbf{m}^{\mathbf{2}} / \mathbf{g}\right)\end{array}$ & $\begin{array}{c}\text { Área microporosa } \\
\left(\mathbf{m}^{\mathbf{2}} / \mathbf{g}\right)\end{array}$ \\
\hline $\mathbf{C A}$ & 905 & 140 & 765 \\
$\mathbf{A C A}$ & 1410 & 138 & 1272 \\
\hline
\end{tabular}

En la figura 1 se observa las micrografías SEM de las muestras CA y ACA. En ambos casos se evidencia la presencia de meso y microporosidad en el material. Se puede apreciar que la muestra ACA, presenta una superficie más irregular y con mayor porosidad en comparación con CA. Por otro lado, se confirma que el carbón activado mantiene su estructura inicial después del tratamiento de oxidación, sin mayores daños en el aspecto morfológico. 

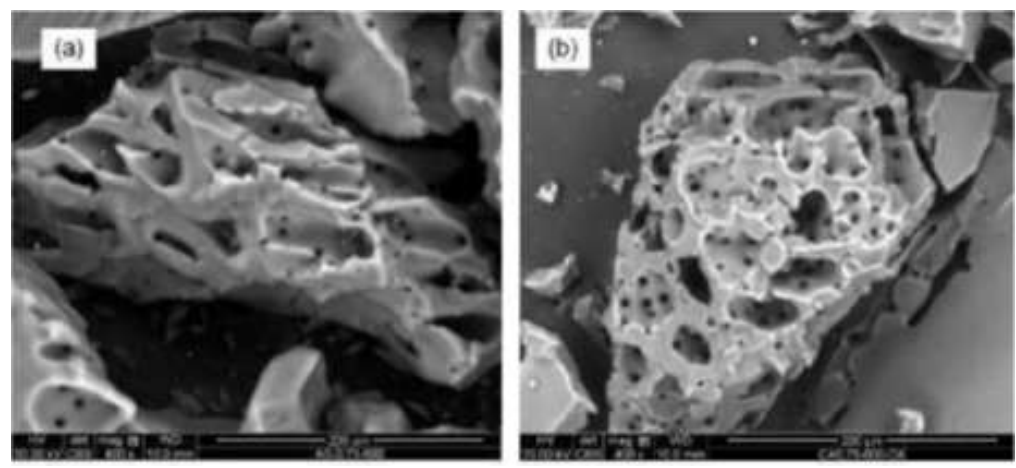

Figura 1. Micrografía SEM de las muestras (a) CA y (b) ACA, con aumento de 400X

La tabla 2 muestra los resultados obtenidos de los grupos oxigenados determinados por Titulación Boehm, así como también los valores del punto de carga cero de los materiales adsorbentes. El carbón activado (CA), muestra una variedad de grupos superficiales oxigenados (carboxílicos, lactónicos y fenólicos). Se puede observar en la muestra ACA que el proceso de oxidación incrementa la acidez total, favoreciendo la formación de grupos fenólicos (acidez débil) y principalmente carboxílicos (acidez medianamente débil), por otro lado, se redujo la presencia de los grupos lactónicos. Esta misma tendencia respecto a los grupos oxigenados, producto de la oxidación con ácido nítrico, se reportó en otros trabajos $^{9,13,14}$. Por otro lado, los resultados del punto de carga cero muestran que ambos carbones tienen una superficie ácida, la cual se incrementa con la oxidación del carbón, este resultado es concordante con los resultados obtenidos con la Titulación Boehm.

Tabla 2. Determinación de los grupos oxigenados superficiales y pHPZC de las muestras CA y ACA

\begin{tabular}{cccccc}
\hline Muestra & $\begin{array}{c}\text { Grupos } \\
\text { carboxílicos } \\
\left(\mathbf{m m o l H ^ { + }} \cdot \mathbf{g}^{-\mathbf{1}}\right)\end{array}$ & $\begin{array}{c}\text { Grupos } \\
\text { lactónicos } \\
\left(\mathbf{m m o l H}^{+} \cdot \mathbf{g}^{-1}\right)\end{array}$ & $\begin{array}{c}\text { Grupos } \\
\text { fenólicos } \\
\left(\mathbf{m m o l H}^{+} \cdot \mathbf{g}^{-1}\right)\end{array}$ & $\begin{array}{c}\text { Grupos Ácidos } \\
\text { Totales } \\
\left(\mathbf{m m o l H}^{+} \cdot \mathbf{g}^{-1}\right)\end{array}$ & pHPZC \\
\hline CA & 1,12 & 3,08 & 0,03 & 4,22 & 2,450 \\
ACA & 4,10 & 0,05 & 0,67 & 4,82 & 2,014 \\
\hline
\end{tabular}

Los espectros FTIR de los carbones activados (figura 2), son consistentes con los valores de acidez total encontrados después del proceso de oxidación. Se puede apreciar un aumento en la intensidad de los picos característicos de los grupos funcionales oxigenados. En los espectros FTIR se aprecian tres bandas de interés: una ubicada a $3425 \mathrm{~cm}^{-1}$, asociada a las vibraciones de estiramiento $\mathrm{O}-\mathrm{H}$ en alcoholes y ácidos carboxílicos. Una segunda banda a $1700 \mathrm{~cm}^{-1}$, de baja intensidad y que se aprecia mejor en la muestra ACA, es asociada a vibraciones de estiramiento de enlaces $\mathrm{C}=\mathrm{O}$ de grupos carbonilos en aldehídos y cetonas, $\mathrm{y}$ la fuerte señal entre 1100-1000 $\mathrm{cm}^{-1}$ atribuida a la presencia de enlaces simples C-O en ácidos carboxílicos. En los tres casos se aprecia un aumento en la intensidad de la señal con respecto al carbón original (CA). En estudios similares con ácido nítrico realizados por Figuereido et al. ${ }^{14}$ también reportaron incrementos de estos grupos oxigenados. 


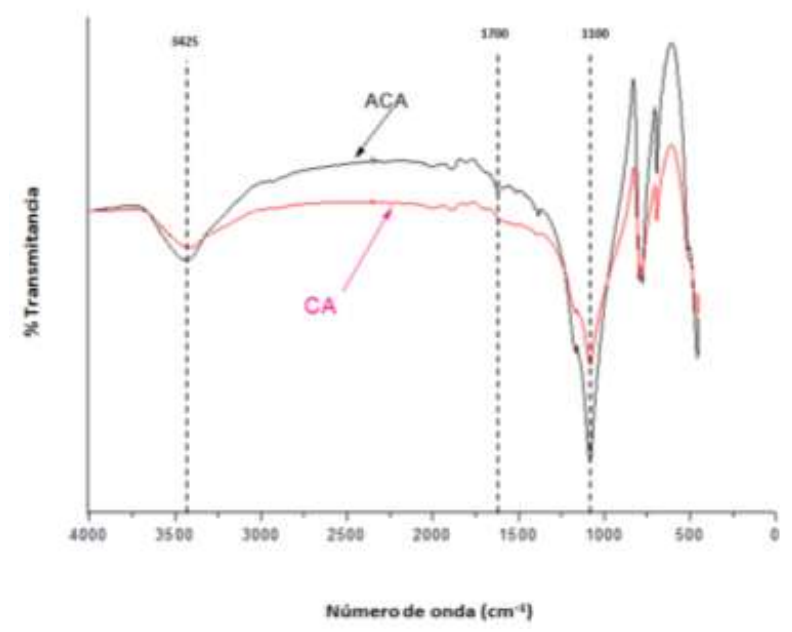

Figura 2. Espectros FTIR de los carbones CA y ACA

\section{Proceso de adsorción}

Se realizaron los ensayos cinéticos para determinar el tiempo de contacto necesario para alcanzar las condiciones de equilibrio. Las pruebas se realizaron a temperatura ambiente, utilizando una concentración inicial de $100 \mathrm{ppm}$ de cada compuesto fenólico (adsorbato), siguiendo el procedimiento descrito anteriormente. En las figuras 3-5 se presentan las curvas cinéticas comparativas de ambos carbones obtenidas para la adsorción del fenol, 4-clorofenol y 4-nitrofenol, respectivamente. Todas las curvas mostradas son cóncavas y alcanzan una estabilidad (condición de equilibrio) en aproximadamente $3 \mathrm{~h}$. Desde el inicio, las curvas se muestran muy cerca del eje qt, lo que indica una fuerte interacción adsorbato-adsorbente, que se evidencia por la mayor cantidad del adsorbato retenido sobre el adsorbente. La adsorción fue mayor para el 4-nitrofenol, seguido de 4-clorofenol y el fenol. Por otro lado, en los tres casos se observa para el carbón ACA, una disminución de la capacidad de adsorción de acuerdo al siguiente orden: fenol $>4$-clorofenol $>$ 4-nitrofenol.

El mecanismo de adsorción involucra la interacción de la densidad electrónica $\pi$ deslocalizados en la superficie del adsorbente proveniente de las láminas de grafeno que componen los carbones CA y ACA, y la densidad electrónica $\pi$ del anillo bencénico en la estructura de cada compuesto fenólico. Esto correspondería a un mecanismo de fisisorción en el cual las especies involucradas se encuentran atraídas mediante fuerzas de van der Waals, es decir por atracciones electrostáticas.

Los grupos funcionales básicos en la superficie del carbón incrementan la densidad electrónica en las láminas de grafeno del carbón activado, de este modo las interacciones $\pi-\pi$ son más fuertes. Este efecto se ve reducido al oxidar el carbón ACA con ácido nítrico, puesto que al incrementarse los grupos oxigenados, principalmente los carboxílicos, estos se ionizan sustancialmente a $\mathrm{pH}$ ácidos produciendo iones $\mathrm{H}^{+}$. Por lo anterior, se dedujo que la 
adsorción de compuestos fenólicos requirió de más grupos básicos y menos grupos ácidos oxigenados, ello explica la baja retención observada en la muestra ACA (figuras 3-5). Este argumento fue también confirmado por el punto de carga cero del carbón ACA (tabla 2).



Figura 3. Cinética de adsorción de fenol sobre las muestras CA y ACA

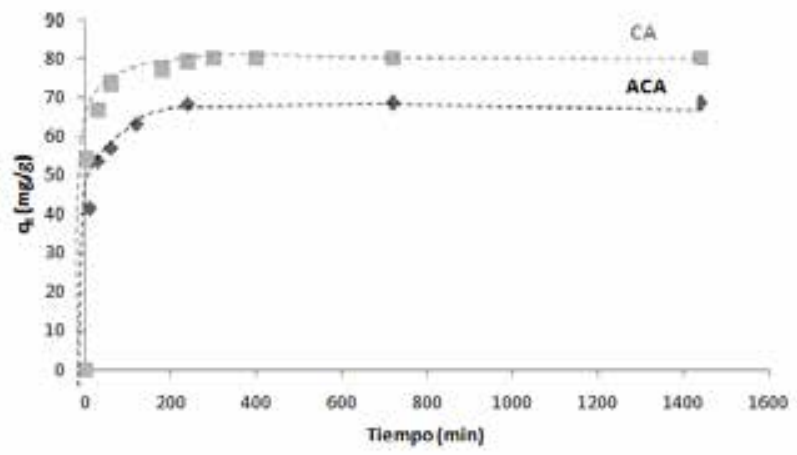

Figura 4. Cinética de adsorción de 4-clorofenol sobre las muestras CA y ACA

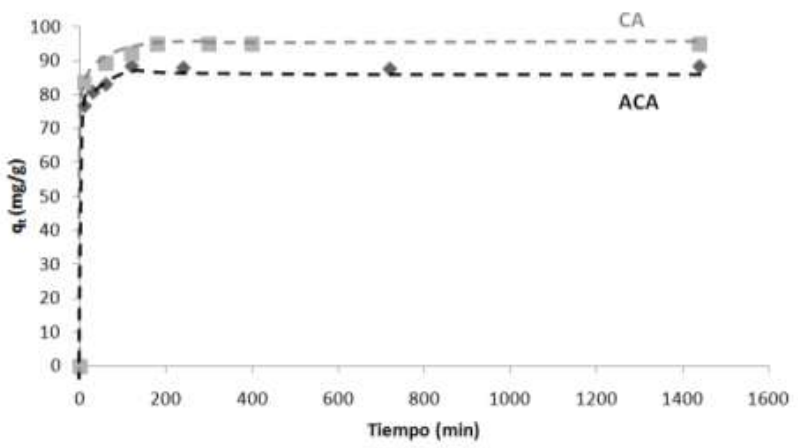

Figura 5. Cinética de adsorción de 4-nitrofenol sobre las muestras CA y ACA 
Las tablas 3 y 4 muestran los parámetros obtenidos al correlacionar los resultados cinéticos con los modelos de pseudo primer orden y pseudo segundo orden. Se observó que con el modelo de pseudo segundo orden se obtuvieron valores del coeficiente de correlación $\left(\mathrm{R}^{2}\right)$ cercanos a 1 y desviaciones estándar normalizadas ( $\Delta$ qe $\%)$ más bajas que con el otro modelo. De igual manera, de las tablas, se puede observar que el orden de adsorción fue el siguiente: 4-nitrofenol > 4-clorofenol $>$ fenol.

Esto es concordante con la teoría de adsorción propuesta por Coughlin y Ezra ${ }^{6}$. El fenol posee solo el grupo $(-\mathrm{OH})$ que es considerado fuertemente activante, lo que disminuye la densidad de carga electrónica en el anillo aromático. El 4-clorofenol contiene al átomo de cloro $(-\mathrm{Cl})$, que es débilmente desactivante y se encuentra en posición antagonista al grupo $(-\mathrm{OH})$ en la molécula, lo que merma la acción de este grupo. El 4-nitrofenol contiene el grupo $\left(-\mathrm{NO}_{2}\right)$ que es fuertemente desactivante y posee la característica de ser atractor de la densidad electrónica, por lo que la carga negativa pudo ser mejor estabilizada, esta se deslocaliza en los átomos de oxígeno o nitrógeno que son fuertemente electronegativos lo que favoreció la adsorción de este compuesto sobre la superficie del carbón CA, pero este efecto se redujo cuando el carbón fue oxidado.

La formación de complejos donor-aceptor (denominado complejo $\pi$ o de transferencia de carga) postulada por otros investigadores 15 podría ser la causa de la adsorción de los compuestos fenólicos sobre el carbón activado en medio acuoso. Se puede comparar este tipo de mecanismo como el de una sustitución electrofílica aromática en química orgánica, donde los adsorbatos actúan como electrófilos y las láminas de grafeno del carbón activado como nucleofilos. La presencia de sustituyentes como el átomo de cloro o el grupo $\left(-\mathrm{NO}_{2}\right)$ en el anillo aromático del fenol, lo vuelve más electrófilo, favoreciendo la adsorción.

Tabla 3. Parámetros obtenidos con el modelo de pseudo primer orden para la adsorción de fenol, 4-clorofenol y 4-nitrofenol sobre los carbones CA y ACA

\begin{tabular}{|c|c|c|c|c|c|c|}
\hline \multirow{2}{*}{$\begin{array}{l}\text { Compuesto } \\
\text { Parámetro }\end{array}$} & \multicolumn{2}{|c|}{ fenol } & \multicolumn{2}{|c|}{ 4-clorofenol } & \multicolumn{2}{|c|}{ 4-nitrofenol } \\
\hline & $\mathrm{CA}$ & $\mathrm{ACA}$ & $\mathrm{CA}$ & ACA & $\mathrm{CA}$ & $\mathrm{ACA}$ \\
\hline $\mathbf{k}_{1}\left(\mathrm{~min}^{-1}\right)$ & 0,011 & 0,023 & 0,029 & 0,019 & 0,031 & 0,019 \\
\hline $\mathrm{q}_{\mathrm{e}}(\mathrm{mg} / \mathrm{g})$ & 17,171 & 23,172 & 62,877 & 43,191 & 45,253 & 19,726 \\
\hline$\Delta q_{e} \%$ & 92,41 & 57,41 & 51,87 & 65,74 & 65,54 & 92,38 \\
\hline $\mathbf{R}^{2}$ & 0,462 & 0,944 & 0,757 & 0,923 & 0,958 & 0,737 \\
\hline
\end{tabular}


Tabla 4. Parámetros obtenidos con el modelo de pseudo segundo orden en la adsorción de fenol, 4-clorofenol y 4-nitrofenol sobre los carbones CA y ACA

\begin{tabular}{|c|c|c|c|c|c|c|}
\hline \multirow{2}{*}{$\begin{array}{l}\text { Compuesto } \\
\text { Parámetro }\end{array}$} & \multicolumn{2}{|c|}{ fenol } & \multicolumn{2}{|c|}{ 4-clorofenol } & \multicolumn{2}{|c|}{ 4-nitrofenol } \\
\hline & $\mathrm{CA}$ & $\overline{\mathrm{ACA}}$ & $\mathrm{CA}$ & ACA & $\mathrm{CA}$ & ACA \\
\hline$k_{2}\left(g \cdot m^{-1} \min ^{-1}\right)$ & 0,003 & 0,003 & 0,002 & 0,002 & 0,004 & 0,005 \\
\hline $\mathrm{q}_{\mathrm{e}}(\mathrm{mg} / \mathrm{g})$ & 66,376 & 37,624 & 81,232 & 69,036 & 95,608 & 88,642 \\
\hline$\Delta q_{e} \%$ & 8,55 & 7,09 & 12,26 & 4,52 & 5,42 & 2,95 \\
\hline $\mathrm{R}^{2}$ & 0,999 & 0,999 & 0,999 & 0,999 & 0,999 & 0,999 \\
\hline
\end{tabular}

Como se observa en la tabla 5 la presencia de los grupos sustituyentes también puede afectar las propiedades físicas y químicas de los compuestos fenólicos. Así, se observó mayor adsorción en los compuestos fenólicos que tienen mayor tamaño y masa molar: 4-nitrofenol $(139,1 \mathrm{~g} / \mathrm{mol})>4$-clorofenol $(128,6 \mathrm{~g} / \mathrm{mol})>$ fenol $(94,1 \mathrm{~g} / \mathrm{mol})$, esto se explica por las interacciones intermoleculares de London, las cuales son directamente proporcionales a la masa molar. Por otro lado, la acidez de los compuestos fenólicos se mide en base a su pKa (tabla 5). Mientras menor sea el pKa mayor será la disociación del ácido y por tanto su fuerza. De la misma manera, el pKa se puede usar cuantitativamente para determinar la proporción o fracción de las especies protonada (o neutra) y desprotonada en diferentes condiciones de $\mathrm{pH}$. Cuando $\mathrm{pH}=\mathrm{pKa}$ la concentración de ambas especies es la misma. Cuando $\mathrm{pH}<\mathrm{pKa}$, la especie que predomina es la protonada. Por el contrario, cuando $\mathrm{pH}>\mathrm{pKa}$ la especie que se encuentra en mayor proporción es la desprotonada. Por lo tanto, dependiendo de la especie que se encuentra en solución, la adsorción se ve favorecida o disminuida.

Adicionalmente, hay una relación inversa entre la adsorción con la solubilidad. En este caso, el compuesto menos soluble (4-nitrofenol) fue el más adsorbido, por el contrario, el más soluble (fenol) fue el menos adsorbido.

Tabla 5. Propiedades de los compuestos fenólicos

\begin{tabular}{ccccc}
\hline $\begin{array}{c}\text { Compuesto } \\
\text { fenólico }\end{array}$ & $\begin{array}{c}\text { Masa molar } \\
\mathbf{( g / m o l})\end{array}$ & $\begin{array}{c}\text { Dimensiones } \\
\text { moleculares }(\mathbf{\AA})\end{array}$ & $\begin{array}{c}\text { Solubilidad } \\
\mathbf{a ~ 2 5}^{\circ} \mathbf{C}(\mathbf{g} / \mathbf{L})\end{array}$ & pKa \\
\hline fenol & 94,1 & $5,76 \times 4,17$ & 93 & 9,89 \\
4-clorofenol & 128,6 & $6,47 \times 4,17$ & 27 & 9,37 \\
4-nitrofenol & 139,1 & $6,84 \times 4,17$ & 16 & 7,08 \\
\hline
\end{tabular}




\section{CONCLUSIONES}

Se logró obtener un carbón activado modificado con ácido nítrico en condiciones no severas de oxidación, con mayor cantidad de grupos funcionales ácidos totales y con mayor porosidad comparada con el carbón activado sin modificar.

La adsorción de los compuestos fenólicos fue principalmente dependiente de la química superficial de los carbones activados. Ambas muestras CA y ACA fueron utilizadas en la adsorción de fenol, 4-clorofenol y 4 nitrofenol. Las curvas cinéticas obtenidas mostraron que la capacidad de adsorción del carbón activado está relacionada con la mayor presencia de electrófilos superficiales en el anillo aromático $(-\mathrm{Cl}$ y $-\mathrm{NO} 2)$, por el mayor tamaño y por la menor solubilidad de la molécula del adsorbato. De acuerdo a la capacidad de adsorción obtenida el orden fue el siguiente: 4-nitrofenol $>4$-clorofenol $>$ fenol.

El carbón modificado presentó una disminución en la capacidad de adsorción, debido a que la oxidación incrementó los grupos oxigenados en la superficie del carbón. La acidez total del carbón oxidado disminuyó de 2,450 $\mathrm{mmolH}^{+}$. $\mathrm{g}^{-1}$ a 2,014 $\mathrm{mmolH}^{+}$. $\mathrm{g}^{-1}$. Los espectros FTIR de los carbones corroboraron el incremento de grupos carboxílicos y fenólicos sobre su superficie.

\section{AGRADECIMIENTO}

Los autores agradecen el apoyo financiero brindado por CONCYTEC a través del Proyecto $\mathrm{N}^{\circ} 12-2013$ FONDECYT vinculado a la Maestría en Química, titulado: Desarrollo de tecnologías adecuadas y de bajo costo para el tratamiento de aguas residuales que contengan colorantes y nitrofenoles. También agradecen al VRI-PUCP y a la Sección Química de la Pontificia Universidad Católica del Perú por el apoyo logístico brindado.

\section{REFERENCIAS BIBLIOGRÁFICAS}

1. Biniak S, Pakula M, Szymanski G, Swiatkowski A. Effect of activated surface oxygenand/or nitrogen-containing groups on adsorption of copper (II) ions from aqueous solution. Langmuir, 1999; 15: 6117-6122.

2. Rivera J, Sánchez M, Gómez V, Álvarez P, Alvim M, Dias J. Activated carbon modification to enhances its water treatment applications. An overview. Journal of Hazardous Materials, 2011; 187: 1-23.

3. Goyal M., Rattan V, Aggarwal D, Bansal R. Removal of cooper from aqueous solution by adsorption on activated carbon. Colloids Surf., 2001; 190: 229-238.

4. Jia Y., Thomas K. Adsorption of cadmium ions on oxygen surface sites in activated carbon. Langmuir, 2000; 16: 1114-1122.

5. Hydar S, Ferro M, Rivera J, Joly J. Adsorption of p-nitrofenol on an activated carbon with different oxidations. Carbon, 2003, 41: 387-395. 
6. Coughlin R, Ezra S. Role of surface acidity in the adsorption of organic pollutants on the surface of carbon. Environ Sci. Technol., 1968; 2: 291-297.

7. Obregon-Valencia D, Sun-Kou M.R. Comparative cadmium adsorption study on activated carbon prepared from aguaje (Mauritia flexuosa L.F.) and olive fruit stones (Olea europaea L.). J. Environ. Chem. Eng., 2014, 2: 2280-2288.

8. Aylas J, Sun-Kou M.R. Estudio de la capacidad adsortiva del carbón activado preparado a partir de semillas de aguaje (Mauritia flexuosa L.F.) en la adsorción de fenol, 4-clorofenol y 4-nitrofenol. En: Actas del II Workshop on Adsorption, Catalysis and Porous Materials. Universidad de los Andes-Bogotá 2013; 105-106.

9. Rodríguez P, Giraldo L, Moreno J. Oxidación de la superficie de carbón activado mediante $\mathrm{HNO}_{3}$ y $\mathrm{H}_{2} \mathrm{O}_{2}$ : Efecto sobre la remoción de níquel (II) en solución acuosa. Revista colombiana de química, 2011; 40(3): 349-364.

10. Chun Y, Mohd K, Wan D. Review of modifications of activated carbon for enhancing contaminant uptakes from aqueous solutions. Sep. Purif. Technol., 2007; 52: 403-415.

11. Rios A, Alves D, Dalmazio I, Fernando S, Bento V, Donnici C, Lago R. Tailoring activated carbon by surface chemical modification with $\mathrm{O}, \mathrm{S}$ and $\mathrm{N}$ containing molecules. Mater.Res., 2003; 2:129-135.

12. Aburub A, Wurster D. Phenobarbital interactions with derivatized activated carbon surfaces. J. Colloid Interf. Sci., 2006; 296: 79-85.

13. Petit C, Peterson G, Mahle J, Bandosz T. The effect of oxidation on the surface chemistry of sulfur-containing carbons and their arsine adsorption capacity. Carbon, 2010; 48: 1779-1787.

14. Figuereido J, Pereira, M, Freitas M, Orfao J. Modification of the surface chemistry of activated carbons. Carbon, 1999; 37:1379-1389.

15. McGuire M, Suffet I. Treatment of water by granular activated carbon. American Chemical Society, 1983; 202: 82-88. 